\title{
FISS Observations of Vertical Motion of Plasma in Tiny Pores
}

\author{
K.-S. Cho • S.-C. Bong • J. Chae • Y.-H. Kim • \\ Y.-D. Park • Y. Katsukawa
}

Received: 20 December 2011 / Accepted: 7 November 2012 / Published online: 15 January 2013

(C) The Author(s) 2013. This article is published with open access at Springerlink.com

\begin{abstract}
Pores can be exploited for the understanding of the interaction between smallscale vertical magnetic field and the surrounding convective motions as well as the transport of mechanical energy into the chromosphere along the magnetic field. For better understanding of the physics of pores, we investigate tiny pores in a new emerging active region (AR11117) that were observed on 26 October 2010 by the Solar Optical Telescope (SOT) on board Hinode and the Fast Imaging Solar Spectrograph (FISS) of the 1.6 meter New Solar Telescope (NST). The pores are compared with nearby small magnetic concentrations (SMCs), which have similar magnetic flux as the pores but do not appear dark. Magnetic flux density and Doppler velocities in the photosphere are estimated by applying the centerof-gravity method to the Hinode/Spectro-Polarimeter data. The line-of-sight motions in the lower chromosphere are determined by applying the bisector method to the wings of the $\mathrm{H} \alpha$
\end{abstract}

Initial Results from FISS

Guest Editor: Jongchul Chae

Electronic supplementary material The online version of this article

(doi:10.1007/s11207-012-0196-1) contains supplementary material, which is available to authorized users.

K.-S. Cho ( $\varangle)$ · S.-C. Bong · Y.-H. Kim · Y.-D. Park

Korea Astronomy and Space Science Institute, Daejeon 305-348, Korea

e-mail: kscho@kasi.re.kr

K.-S. Cho

NASA Goddard Space Flight Center, Greenbelt, MD 20771, USA

K.-S. Cho

Department of Physics, The Catholic University of America, Washington, DC 20064, USA

J. Chae

Astronomy Program, Department of Physics and Astronomy, Seoul National University, Seoul 151-747,

Korea

e-mail: chae@astro.snu.ac.kr

Y. Katsukawa

National Astronomical Observatory of Japan, 2-21-1 Osawa, Mitaka, Tokyo 181-8588, Japan

e-mail: yukio.katsukawa@nao.ac.jp 
and the Ca II $8542 \AA$ line simultaneously taken by the FISS. The coordinated observation reveals that the pores are filled with plasma which moves down slowly and are surrounded by stronger downflow in the photosphere. In the lower chromosphere, we found that the plasma flows upwards inside the pores while the plasma in the SMCs is always moving down. Our inspection of the Ca II $8542 \AA$ line from the wing to the core shows that the upflow in the pores slows down with height and turns into downflow in the upper chromosphere while the downflow in the SMCs gains its speed. Our results are in agreement with the numerical studies which suggest that rapid cooling of the interior of the pores drives a strong downflow, which collides with the dense lower layer below and rebounds into an upflow.

Keywords Chromosphere $\cdot$ Imaging spectroscopy $\cdot$ Photosphere $\cdot$ Pore $\cdot$ Surface magnetic field

\section{Introduction}

While sunspots can grow to tens of thousands of kilometers, pores are patches of strong magnetic field only about a few Mm wide at the photosphere without penumbral structures. Their simple magnetic field configuration allows investigation of the dynamic interaction between solar magnetic elements and granular convection, and of the transport of energy by flows or waves along strong magnetic field to the chromosphere and corona.

The photospheric appearance of pores has been investigated using ground- and spacebased observations. High resolution observations with ground-based telescopes reveal that pores

i) are magnetized areas whose (magnetic) radius is larger than its continuum radius (Keppens and Martinez Pillet, 1996),

ii) are structured with fine structures (e.g. an umbral dot and a light bridge),

iii) develop/decay by the accumulation and dispersion of surrounding magnetic knots (e.g.

Parker, 1979; Hirzberger, 2003), and

iv) are surrounded by downflow channels (Keil et al., 1999).

Recently, Hinode/SOT observation by Cho et al. (2010) confirmed that the plasma in tiny pores $\left(R<2^{\prime \prime}\right)$ is always moving down, with speeds between 100 and $500 \mathrm{~m} \mathrm{~s}^{-1}$. They found that the darkness increases as the surrounding plasma flows toward them and decreases as the plasma moves away from them. The plasma in the tiny pores in the photosphere is always moving down and the pores are surrounded by a strong neighboring downward flow (highly red-shifted). There are several numerical simulations of magnetic flux tubes in the solar photosphere. For example, Cameron et al. (2007) and Knölker and Schüssler (1988) predicted downflows at the pores. Steiner et al. (1998) and Kato et al. (2011) carried out numerical simulations of dynamic interaction between magnetic flux tubes and the surrounding gas motion. Especially, Kato et al. (2011) reported that the convective downdrafts in the surroundings of a magnetic element in the photosphere leads to a slow and upwardly propagating magnetoacoustic wave, which develops into a shock wave in the chromospheric layers of the magnetic flux tube. Meanwhile, very few chromospheric observations above the pores and small magnetic concentrations (SMCs) have been made, since observations of chromospheric manifestations of pores/SMCs are difficult. This is a major impediment to the understanding of the physics of pores.

The Fast Imaging Solar Spectrograph (FISS; Ahn et al., 2008; Chae et al., 2012) in the Coudé room of the $1.6 \mathrm{~m}$ New Solar Telescope (NST; Goode et al. 2003, 2010) at Big Bear Solar Observatory is a unique system that can perform fast-scan spectroscopy of 
extended areas. Since the NST/FISS is suited to improve the current understanding of the dynamics above the pores/SMCs, we have undertaken a coordinated observation with the Solar Optical Telescope (SOT; Tsuneta et al., 2008), on board the Hinode satellite (Kosugi et al., 2007). Our observation target was a new emerging active region (AR11117) on 26 October 2010.

\section{Observation and Analysis}

A new emerging active region (AR11117) appeared from the east limb on 19 October 2010 with a single stable round sunspot. As the sunspot passed across the disk, a chain of tiny sunspots at the south-east of the main sunspot emerged and became much larger and stretched. When the sunspots were born, they had both a preceding sunspot to the west and a following sunspot groups to the east. Figure 1(a) shows the G-band image of the region taken with Hinode/SOT. Contours in Figure 1(b) indicate the line-of-sight magnetic fields observed with Helioseismic and Magnetic Imager (HMI; Schou et al., 2012) onboard the Solar Dynamic Observatory (SDO; Pesnell, Thompson, and Chamberlin, 2011) superposed on the continuum image from SDO/HMI. It can be seen that the preceding sunspot had a negative polarity and the following sunspot groups had a positive polarity. The pores and SMCs had a negative magnetic field, and they were located between the preceding sunspot and the following sunspot groups as marked by the small box in Figure 1(b).

We have carried out a joint observation of AR11117 with NST/FISS and Hinode/SOT from 25 October to 29 October 2010. The photospheric observation of the pores was mainly done by Hinode/SOT. G-band and Ca II H-line (3968 ̊) images were obtained with the Broad-band Filter Imager (BFI) of Hinode/SOT with a pixel size of $0^{\prime \prime} .16$ and $30 \mathrm{~s}$ time cadence for a field of view (FOV) of $75^{\prime \prime} \times 75^{\prime \prime}$. Basic data processing was performed by using the SolarSoft package (http://www.Imsal.com/solarsoft). G-band images were aligned to track the pores and SMCs by using the local correlation tracking technique, and the Ca II images were shifted by the same amount to align them with simultaneous G-band images by assuming the same pointing of the G-band and Ca II images by the BFI. The SpectroPolarimeter (SP) of Hinode/SOT recorded the magnetically sensitive Fe I 6301.5/6302.5 lines with a spectral resolution of $\approx 21 \mathrm{~m} \AA$. The SP mapped the region with a normal mode raster scan of 512 steps and time cadence of $\approx 50 \mathrm{~min}$. The effective pixel size was $0^{\prime \prime} .16$ along the slit and $0^{\prime \prime} .15$ across it. The field of view was about $75^{\prime \prime} \times 81^{\prime \prime}$. Dark, flat, and wavelength corrections on the SP data were performed by using the SolarSoft package. We have derived line-of-sight magnetic field strengths and line-of-sight velocities from the SP data by using the center-of-gravity (COG) method of Rees and Semel (1979). The SP scans were aligned with BFI images by over-plotting SP continuum data on G-band images.

For the observation of the lower chromosphere, we ran NST/FISS during 17-19 UT from 25 October to 29 October. Our observation target was the pores near AR11117. The seeing at the Big Bear Observatory was fairly good on 26 October so that we selected FISS data taken on that day for the present study. FISS scanned the region including the pores located in the small box of Figure 1(a). The FISS obtained raster-scanned spectral images in two spectral lines, H $\alpha$ and Ca II $8542 \AA$, simultaneously. The FISS mapped the region with the default mode in which the scanner moves one step, waits for each camera to take one frame, and then moves to the next step. Each step took $0.15 \mathrm{~s}$ and the slit was moved over 400 steps. The raster cadence was about $63 \mathrm{~s}$. The effective pixel size was $0^{\prime \prime} .16$ along and across the slit. The number of pixels along the slit was about 256 so that the field of view was about $40^{\prime \prime}$ in the slit direction. The scan width was about $64^{\prime \prime}$. The spectral resolution 

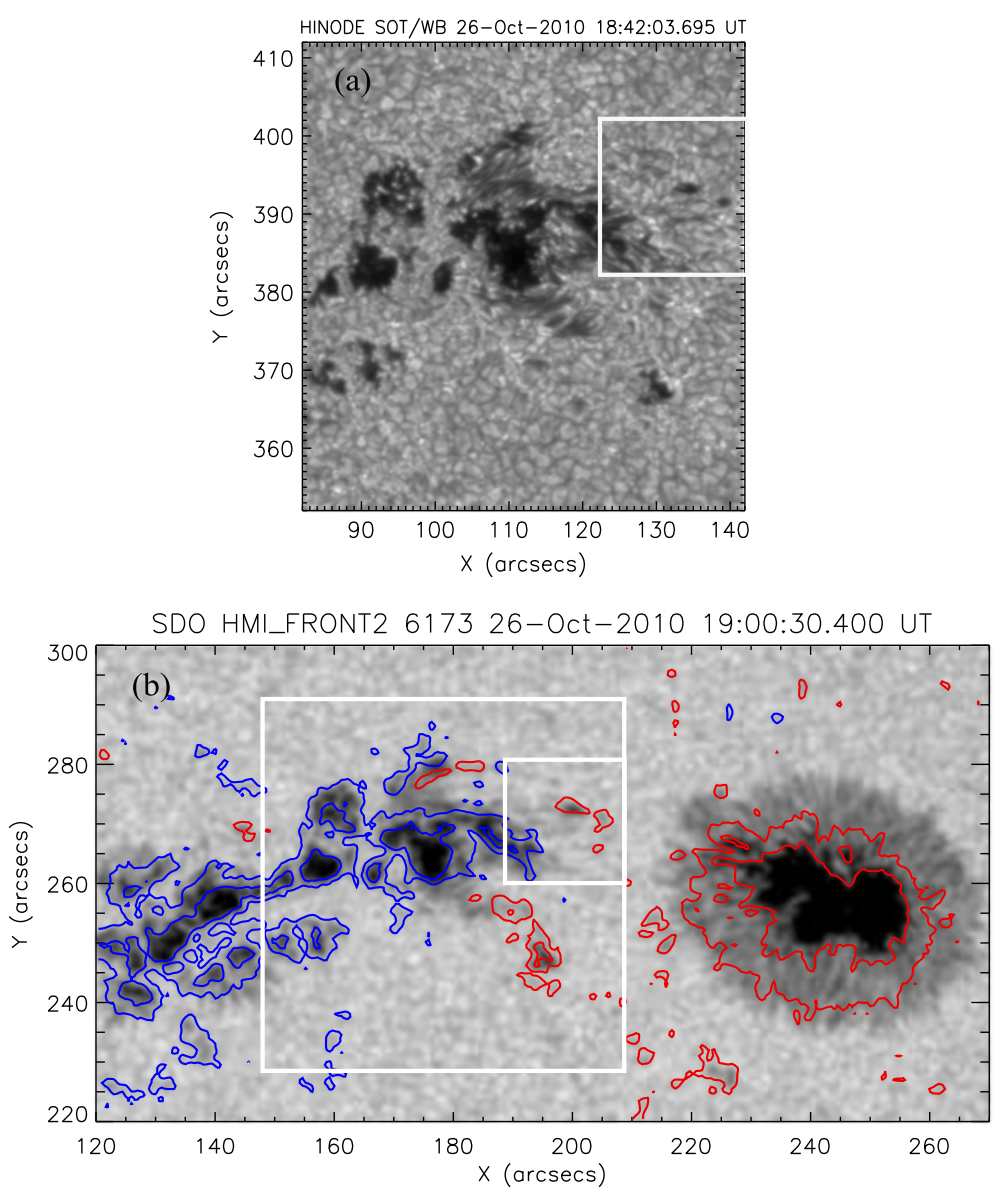

Figure 1 (a) G-band image of a new emerging active region (AR11117) taken with Hinode/SOT at 18:42 UT and (b) SDO/HMI continuum image taken at 19:00 UT on 26 October 2010. AR11117 was located near the disk center (N22W16). The large box in the lower panel denotes the field of view of Hinode/SOT, and the small box in both panels denotes the region of our interest. The contours in panel (b) indicate the line-of-sight magnetic fields obtained with SDO/HMI. Blue and red contours denote the boundary of positive and negative magnetic fields with their strength levels of $400 \mathrm{G}$ and $1000 \mathrm{G}$. There was a data gap in SDO observation between 16:13 UT and 19:00 UT.

of FISS is approximately $19 \mathrm{~m} \AA$ and $21 \mathrm{~m} \AA$ in $\mathrm{H} \alpha$ and Ca II $8542 \AA$, respectively. Details of basic processing including data acquisition, flat fielding, correction, and compression are described by Chae et al. (2012). For wavelength calibration of the spectral lines, we used the two telluric lines of water vapor in the vicinity of each line $(6561.097 / 6565.545 \AA$ for $\mathrm{H} \alpha, 8540.817 / 8546.222 \AA$ for Ca II).

As shown in Figure 2, chromospheric features are dominant in the line-center images, while the outer wings gradually sample the photosphere. This means that the images at different wavelengths from the wing to the center of the $\mathrm{H} \alpha$ and $\mathrm{Ca}$ II 8542 A lines show different features from the photosphere to the chromosphere. It looks likely that the pores and SMCs were covered by fibrils. Both lines showed fibrils with bright feet. Generally, darker $\mathrm{H} \alpha$ fibrils correspond to higher density, and $\mathrm{Ca}$ II brightness is more related to temperature (Cauzzi et al., 2009). 

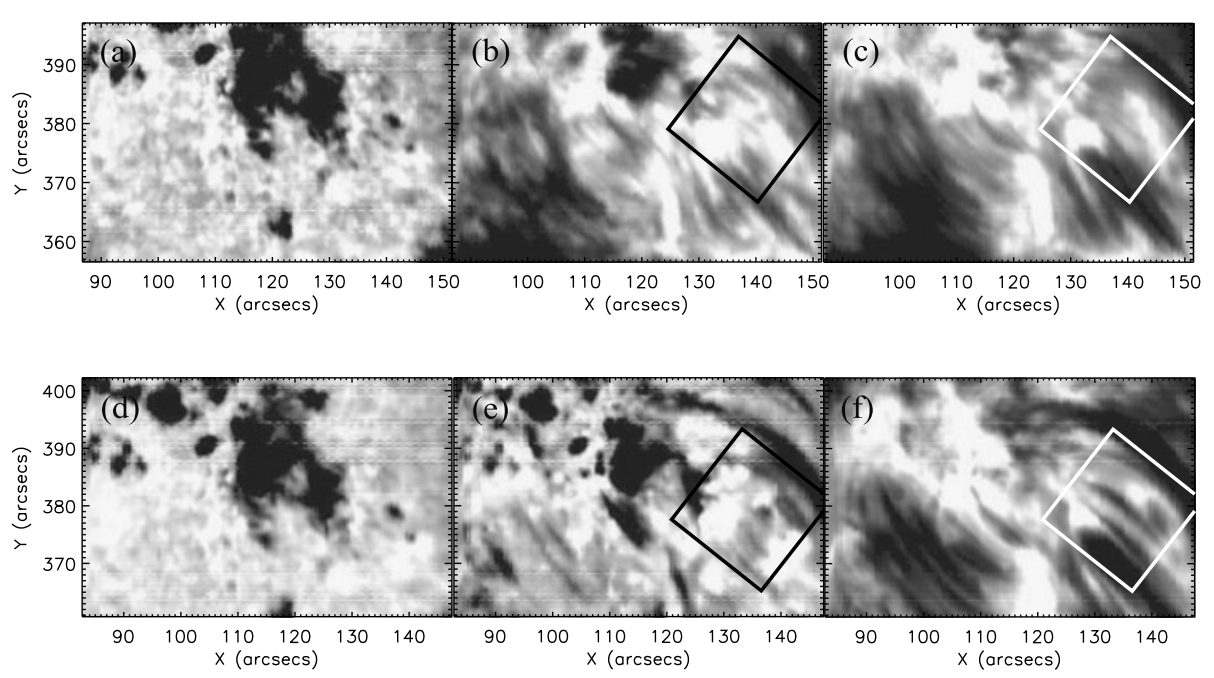

Figure 2 FISS images obtained at $18: 41$ UT on 26 October 2010 at (a) the wing $(-2.5 \AA)$, (b) near the core $(-0.2 \AA)$, and (c) the center of the Ca II $8542 \AA$ line, and (d) the wing $(-2.0 \AA)$, (e) near the core $(-0.7 \AA)$, and (f) the center of the H $\alpha$ line, respectively. The field of view is about $64^{\prime \prime} \times 40^{\prime \prime}$ and the box in each panel denotes the ROI.

\section{Results}

\subsection{General Characteristics of the Pore and SMC}

Figure 3 shows the observation by Hinode/SOT for the ROI which is marked with a small box in Figure 1(a). A part of the penumbra of the following sunspot with positive polarity in AR11117 can be seen. The pores and SMCs were located at the network near the penumbra of the following sunspot in AR11117 at the disk center. As shown in Figure 3(b), we can identify four strong magnetic field areas with negative polarity as denoted by white plus symbols. As shown in the G-band image of Figure 1(a), two larger magnetic regions correspond to dark features (pores; P1 and P2), while the other two regions (SMCs; S1 and S2) with strong magnetic flux do not show any dark features.

Why do not the SMCs appear dark in the G-band image even though they are similar to the pores in magnetic flux density? A standard explanation by Spruit and Zwaan (1981) is that pores as well as sunspots are dark by inhibition of convection, while smaller flux tubes such as SMCs may not necessarily be dark because of radiative energy input from their hot walls into the evacuated interior. For a similar reason, the filling fraction of magnetic field in a single pixel was proposed as an alternative explanation of the difference between pores and SMCs (Knölker and Schüssler, 1988). Morinaga et al. (2008) reported that the filling factor of pores $(0.8-0.9)$ is bigger than that of G-band bright points (0.6). We have estimated the mean filling factor values inside our pores by applying the Milne-Eddington (ME) inversion method (Skumanichi and Lites, 1987) to our SP data. As a result, we found that the filling factor in pores $(0.85$ for $\mathrm{P} 1$ and 0.84 for $\mathrm{P} 2)$ is a little bigger than that of SMCs $(0.81$ for SMC1 and 0.77 for SMC2), being in agreement with these studies.

The line-of-sight velocity in the photosphere deduced from SP data shown in Figure 3 suggests that the tiny pores are surrounded by strong downflows, and are filled with weak downflows inside them. This is consistent with the results of Cho et al. (2010), who suggested that slow downflows inside pores and fast downflows outside may be associated 

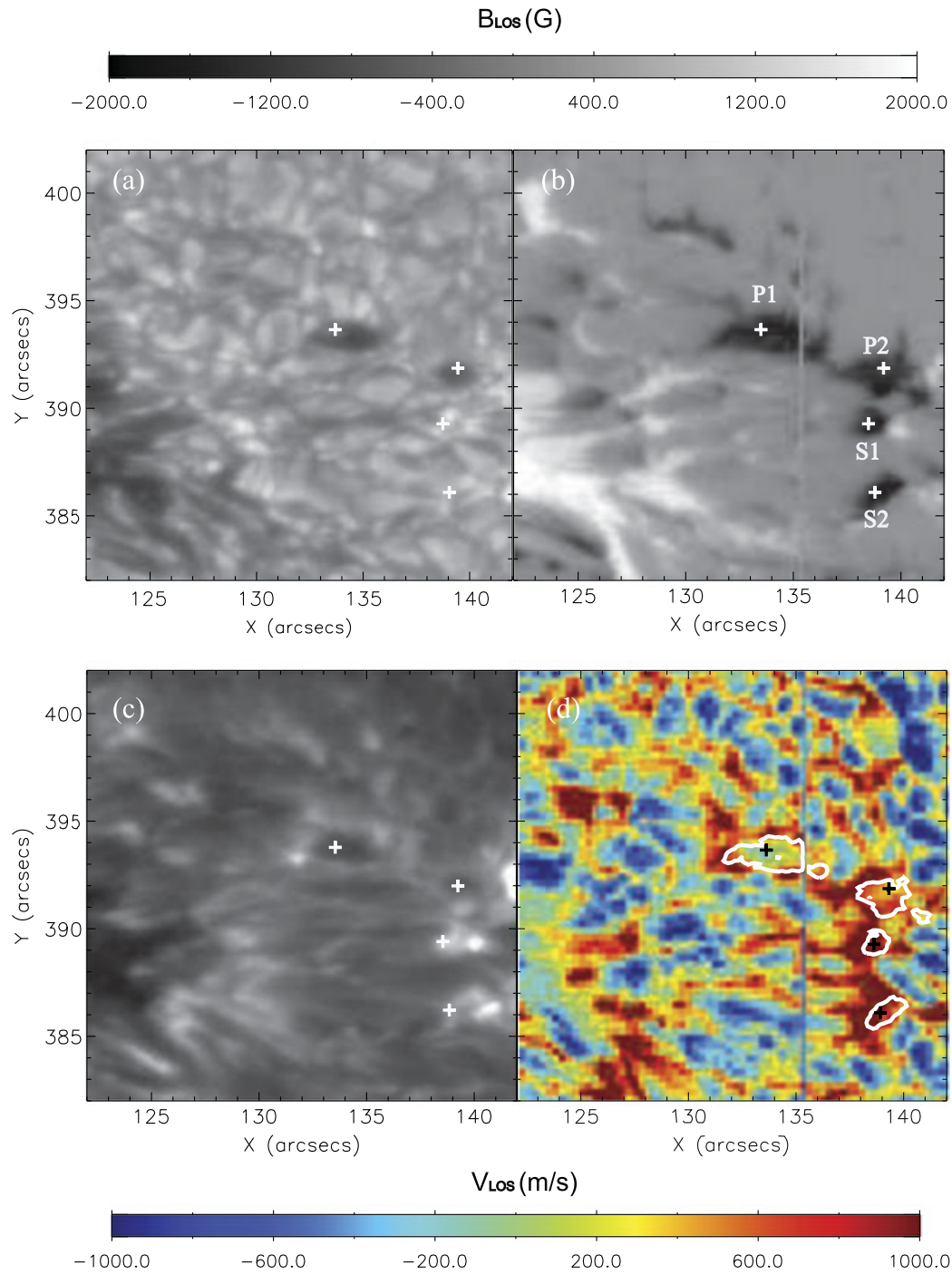

Figure 3 Hinode/SOT observations of (a) BFI G-band, (b) SP line-of-sight magnetic field, (c) BFI Ca II H-line core intensity, and (d) SP Doppler map (red color means downdraft) of ROI specified by the small box in Figure 2. Plus symbols represent the pores and SMCs studied here. The white contour in the Doppler map (d) marks the boundaries of strong magnetic field patches $(1500 \mathrm{G})$ in panel (b).

with the formation and maintenance of pores. The downflow channels around the pores and SMCs were well known from previous observations (e.g. Keil et al., 1999; Nagata et al., 2008; Morinaga et al., 2008) and from numerical simulations (Cameron et al., 2007; Spruit, 1979).

The pores considered in this study are fairly small (diameter $<2^{\prime \prime}$ ). The time evolution of tiny pores may be different from ordinary pores so that it might be interesting to investigate how these intense magnetic structures evolve during our observation. Unfortunately, there were no SDO/HMI data and Hinode/SOT Narrow-band Filter Imager data during our obser- 

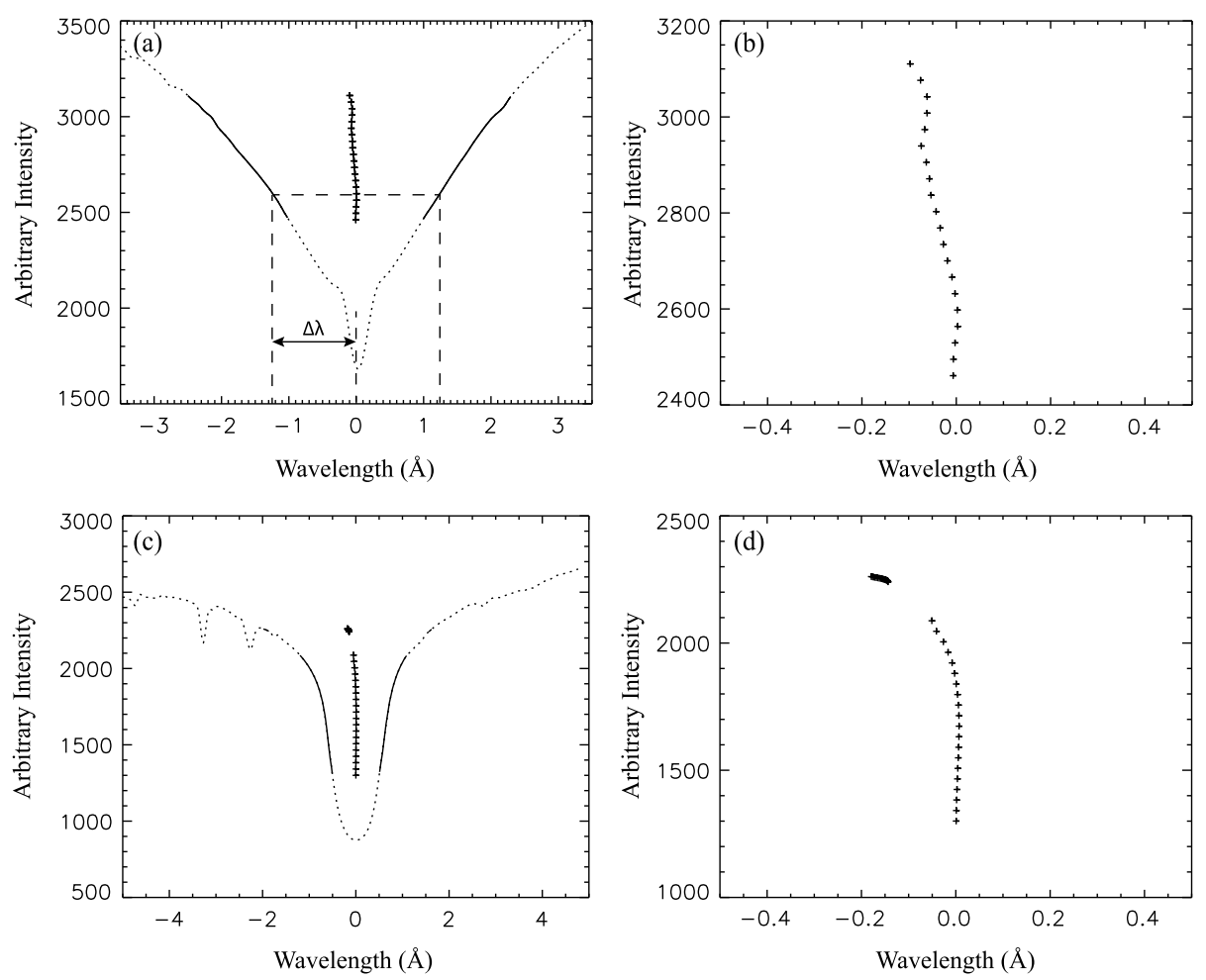

Figure 4 (a) FISS Ca II $8542 \AA$ and (c) H $\alpha$ line profiles averaged over the FISS FOV. Plus symbols denote the bisector in the wavelength ranges (solid lines) that are used for estimation of the line-of-sight velocity. Enlarged views of the bisector are given in (b) Ca II $8542 \AA$ and (d) H $\alpha$. The intensity level for estimation of the bisector is determined at the wavelength offset $(\Delta \lambda)$ as shown in panel (a).

vation period, so we could not directly inspect the evolution of the magnetic field. However, we examined the evolution of the pores by using Hinode/SOT-BFI G-band observation. The G-band movie (electronic supplementary material) shows that the pores underwent temporal change in intensity and size, but they did not significantly grow or decay during the observation.

\subsection{Vertical Motion in the Lower Chromosphere}

The $\mathrm{H} \alpha$ line is formed either in chromospheric fibrils or in deep photosphere whereas the Ca II $8542 \AA$ line samples the layers in between the fibrils and the photosphere (Cauzzi et al. 2008, 2009). Leenaarts et al. (2006) reported that the intensity in the far wings of the Ca II $8542 \AA$ line is highly sensitive to the presence of magnetic structures. In this study, we determined the line-of-sight velocity by applying the bisector measurement to the line profiles of $\mathrm{H} \alpha$ and Ca II $8542 \AA$ acquired by FISS. The line profiles of Ca II $8542 \AA$ (Figure 4(a)) and $\mathrm{H} \alpha$ (Figure 4(b)) are spatially averaged over the FISS FOV as shown in Figure 2. To get the line profile, we applied wavelength correction using the telluric lines as described in the previous section, and then smoothed the spectrum with a $0.1 \AA$ window. The bisector was applied to the wing spectra of the Ca II $8542 \AA$ and H $\alpha$ lines, respectively. The dotted lines in Figures 4(b) and 4(d) exhibit curved inverse-C-shaped bisectors, which are 


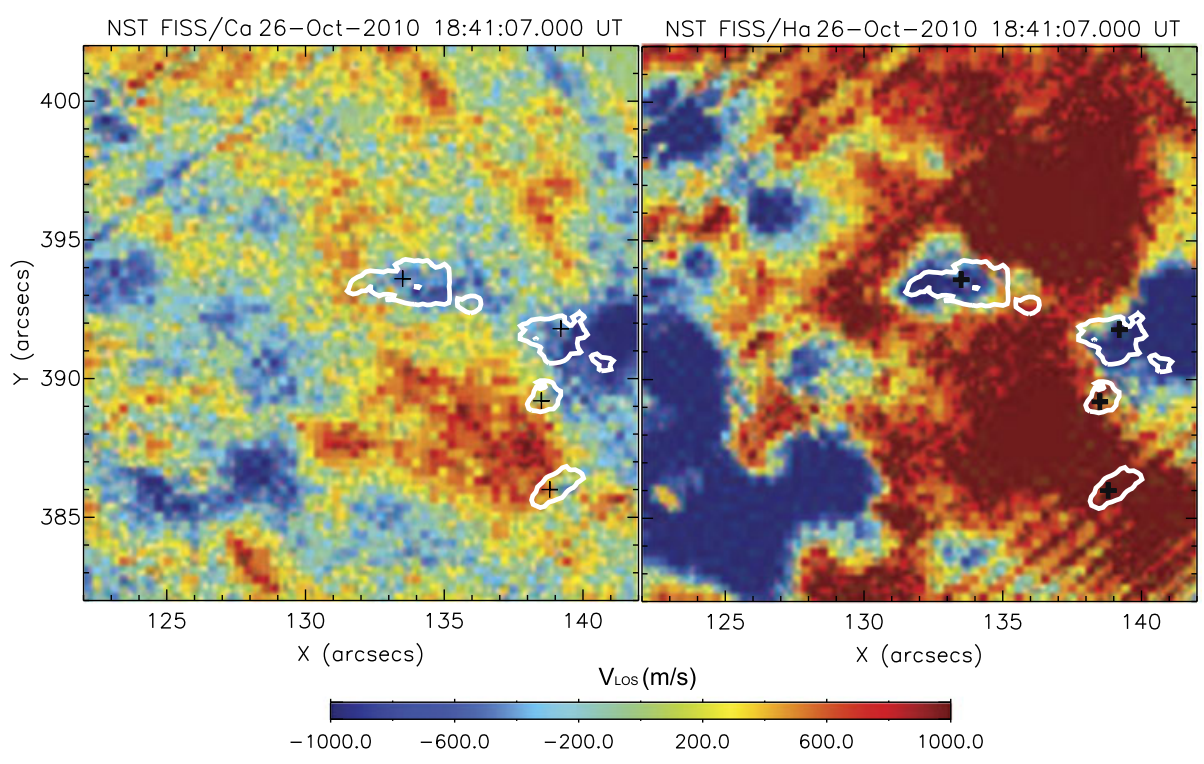

Figure 5 FISS Doppler maps (bisector shift from the nominal line-center wavelength) in the wings of Ca II $8542 \AA$ (left panel, $\Delta \lambda=2.3 \AA$ ) and $\mathrm{H} \alpha$ (right panel, $\Delta \lambda=2 \AA$ ), which are taken at 18:41 UT on 26 October 2010. Plus symbols and white contours are the same as those in Figure 3.

in contrast to the regular C-shape of photospheric spectral line bisectors. On the origin of the inverse-C-shape, Uitenbroek (2006) proposed a possibility that it might be the result of upwardly traveling acoustic waves. It should be noted that the inverse-C-shape which was investigated by Uitenbroek (2006) was from the line core of the Ca II $8542 \AA$ line, while our inverse-C-shape is from the wing of the Ca II $8542 \AA$ line.

Since the intensity level in the line spectra reflects the formation height as shown in Figure 2 (Cauzzi et al. 2008, 2009), the bisectors measured from the wings of the line represent the Doppler shift at a lower altitude of the atmosphere. In this study, we considered the wavelength ranges of $\Delta \lambda=1.0-2.5 \AA$ in Ca II $8542 \AA$, and $\Delta \lambda=1.9 \AA$ and $\Delta \lambda=0.5-$ $1.2 \AA$ in $\mathrm{H} \alpha$, respectively.

Figure 5 presents line-of-sight (LOS) velocity maps constructed by using the bisectors at the wings of Ca II $8542 \AA$ (left) and $\mathrm{H} \alpha$ (right). To obtain the H $\alpha$ Doppler map, we applied the bisector method to the spectral intensity level near $\Delta \lambda=2 \AA$ at each pixel and subtracted the reference velocity which is from the spectrum averaged over the whole scan region and added the disk-center average photosphere value $\left(-120 \mathrm{~m} \mathrm{~s}^{-1}\right)$ given by Martinez Pillet, Lites, and Skumanich (1997). Since one of the purposes of this study is to inspect the LOS velocity change with height (wavelength) from the photosphere to the chromosphere by comparing Hinode/SP and NST FISS observations, we need a reference LOS velocity. We can use the spatially averaged LOS velocity in the chromosphere as the reference velocity, but the averaged velocity in the chromosphere may change with time. It is well known that the granule is on average blue-shifted, so we adopted the disk-center average photosphere value $\left(-120 \mathrm{~m} \mathrm{~s}^{-1}\right)$ given by Martinez Pillet, Lites, and Skumanich (1997). The Ca II $8542 \AA$ Doppler map was similarly obtained by using the off-band intensities near $\Delta \lambda=2.3 \AA$. Both maps represent the line-of-sight velocity at 
a lower atmosphere above the photosphere and show similar velocity distribution in general.

As shown in Figure 5, the penumbra shows the blueshift (upflows), while most of the quiet region has redshift (downflows). Pores and SMCs are located at the boundary of the redshift cells. The white contours in Figure 5 showing the level of 1500 gauss $(\mathrm{G})$ delineate the boundaries of strong magnetic patches obtained by Hinode/SP observation, of which spatial resolution is about $0^{\prime \prime} .2$. The effective spatial resolution of the FISS map is about $1^{\prime \prime}$ (Chae et al., 2012), which is similar to the seeing limit at BBSO; therefore the accuracy of the alignment between the NST/FISS and Hinode/SOT is similar to the resolution of the FISS map. As compared with the Hinode/SP Doppler map in Figure 3(d), Figure 5 shows structures larger in scale and therefore the characteristic scales of the velocity field look different. This may be because granule-size photospheric features seen by Hinode/SP disappear in the lower chromosphere, and/or the spatial resolution or sensitivity of NST/FISS is lower than that of Hinode/SP. In both images, we found upflows on the pores and downflows at the SMCs. The upflow areas at the location of the pores in Figure 5 look larger than the photospheric size of pores in the G-band image (Figure 3(a)) and they are slightly shifted in position relative to the pores. It may be related to the expansion of the flux tube, or swaying of it by the ambient horizontal flows in the upper atmosphere.

We inspected the line-of-sight velocity change across the wavelength of the line. As a result, we found that the velocity changes with wavelength in Ca II $8542 \AA$ but this is not the case in $\mathrm{H} \alpha$. This is because the $\mathrm{H} \alpha$ line is formed either in chromospheric fibrils or in the deep photosphere whereas the Ca II $8542 \AA$ line samples the layer between them (Cauzzi et al. 2008, 2009). We speculate that the velocity changes with wavelength seen in Ca II $8542 \AA$ may reflect an intrinsic property of the vertical flows above the pores/SMCs in the lower chromosphere.

Since we are interested in changes in the flow motion with height above the pores in the lower chromosphere, we investigated the Ca II $8542 \AA$ line in detail. Figure 6 shows the line-of-sight velocity changes with wavelength from core $(\Delta \lambda=1.25 \AA)$ to the wing $(\Delta \lambda=2.35 \AA)$ of Ca II $8542 \AA$ line. Unlike Figure 5 , we subtracted the Doppler velocity averaged over weakly magnetized regions in ROI, since our reference velocity set to the quiet photosphere value at disk center given by Martinez Pillet, Lites, and Skumanich (1997) was not convenient. As a result, the Doppler velocity map looks a little different, and the blueshift on the pores is more prominent in the line-wing image taken at $\Delta \lambda=2.35 \AA$ as shown in Figure 6(d). We found that redshift is dominant at the line core (Figure 6(a)), while blueshift is dominant at the wing (Figure 6(d)) in general. The red-shifted cells in the penumbra and near the pore and SMC regions reduce their size as one moves to the far-wing wavelengths. If we accept that the bisector reflects the height variation of the Doppler shift, the downflow in the pores turn to the upflow, and the downflow in the SMCs gets slower as one moves from the upper to the lower chromosphere.

Figure 7 presents the line-of-sight velocity change in the pores and SMCs with wavelength. We measured the mean velocities in the circles in Figure 6 at the pores and SMCs as well as in the weak $(<100 \mathrm{G})$, intermediate, and strong $(>1200 \mathrm{G})$ magnetic field regions in the ROI. As shown in Figure 7, the velocity in pore P1 (P2) changes from $-700 \mathrm{~m} \mathrm{~s}^{-1}$ $\left(-800 \mathrm{~m} \mathrm{~s}^{-1}\right)$ at $\Delta \lambda=2.3 \AA$ to $600 \mathrm{~m} \mathrm{~s}^{-1}\left(5 \mathrm{~m} \mathrm{~s}^{-1}\right)$ at $\Delta \lambda=1.2 \AA$, namely, increasing with height. The velocity in the SMCs also shows similar change with height but in the opposite sign (red-shifted values). It is also found that the flow velocity in the strong magnetic field regions changes from blueshift to redshift while the flow velocity in the weak and intermediate field regions does not change significantly with height. 

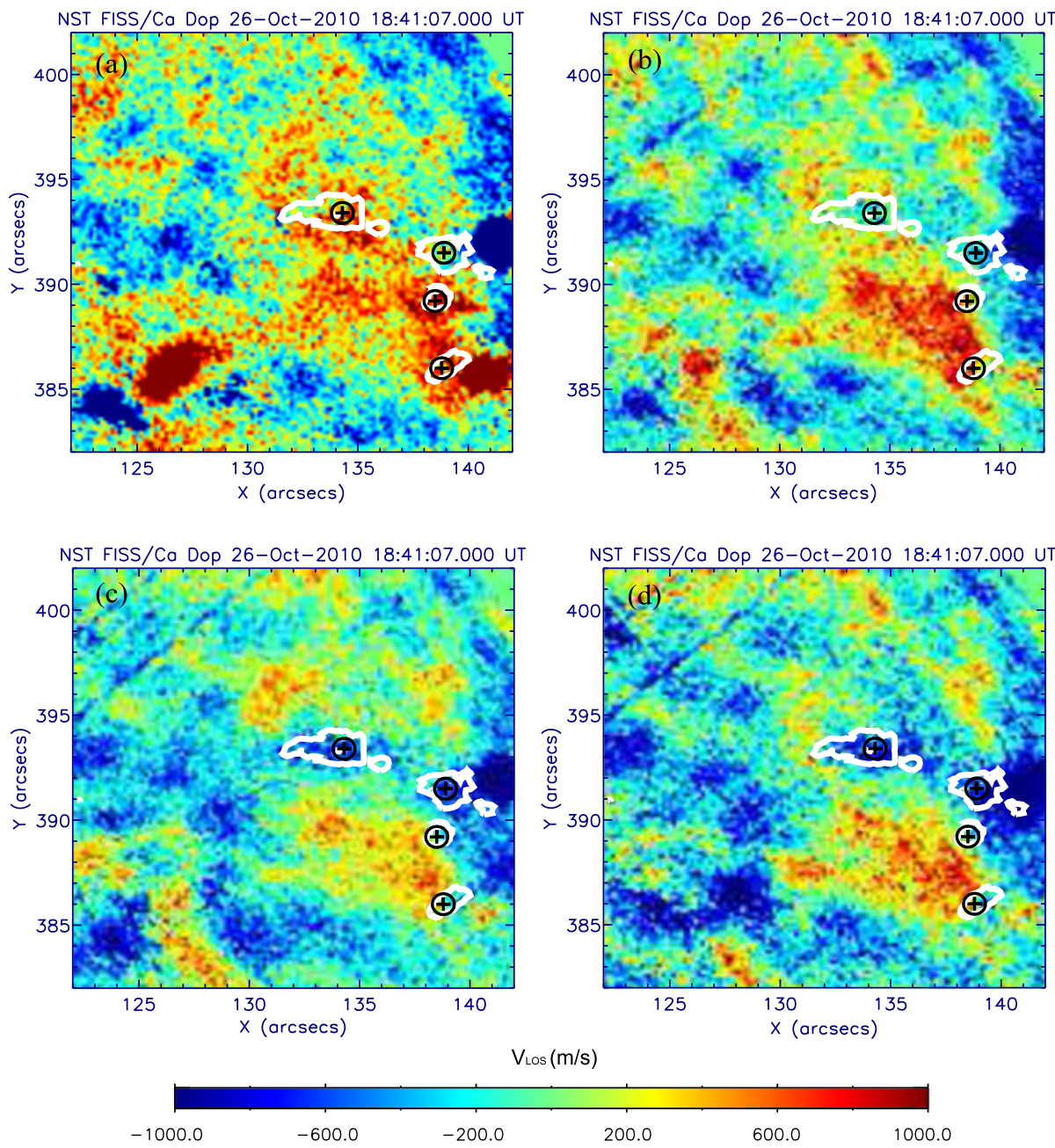

Figure 6 Ca II line-of-sight velocity maps at 18:41 UT on 26 October 2010 at the wavelengths of (a) $\Delta \lambda=1.25 \AA$, (b) $\Delta \lambda=1.55 \AA$, (c) $\Delta \lambda=1.95 \AA$, and (d) $\Delta \lambda=2.35 \AA$. The fringes in panels (c) and (d) are due to data contamination by dust in the FISS observation.

\subsection{Evolution of the Vertical Motion}

We inspected temporal variations of LOS velocities in the pores and SMCs during the FISS observation period (26 October 2010, 18:30 - 18:57 UT). Figure 8 shows the time variations of the LOS velocities above pore P1 that were estimated at different wavelengths from the line wing to the core. Note that the wavelength-dependence of the LOS velocity as shown in Figure 7 was maintained throughout the observing time; for example, LOS velocities at $\Delta \lambda=2.35 \AA$ remained significantly negative and those at $\Delta \lambda=1.25 \AA$ remained significantly positive. The LOS velocities in fact changed with time, either slowly varying or oscillating, but such changes were not drastic enough to alter the pattern described above. We obtained similar results for the other pore (P2) and the two SMCs. This characteristic of velocity field in the low chromosphere of the pores and SMCs we found is similar to that of 


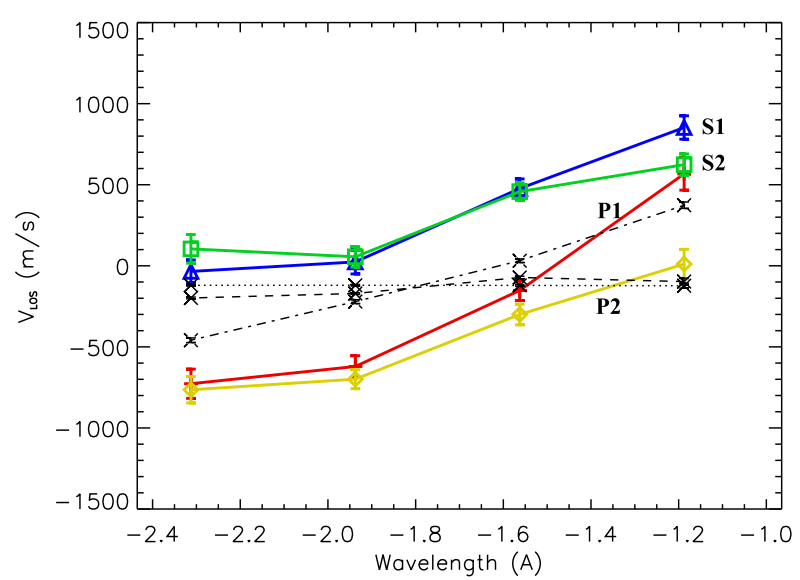

Figure 7 Variation of the line-of-sight velocity in the pores (P1, P2) and SMCs (S1, S2) at various wavelengths from the wing to the core in the Ca II line. Black lines denote the distribution at the strong (dash dotted), intermediate (dashed), and weak (dotted) magnetic field regions. The uncertainty in the mean velocity was estimated from the velocity fluctuation inside the averaged region. The estimated uncertainties are $\approx 100 \mathrm{~m} \mathrm{~s}^{-1}$ for the pores and SMCs, and $\approx 10 \mathrm{~m} \mathrm{~s}^{-1}$ for the strong, intermediate, and weak magnetic regions.

Figure 8 Time variation of the line-of-sight velocity in pore P1 at the wavelengths of

$\Delta \lambda=1.25 \AA$ (thin gray),

$\Delta \lambda=1.55 \AA$ (thick gray),

$\Delta \lambda=1.95 \AA$ (thin black), and

$\Delta \lambda=2.35 \AA$ (thick black) in the

Ca II line.

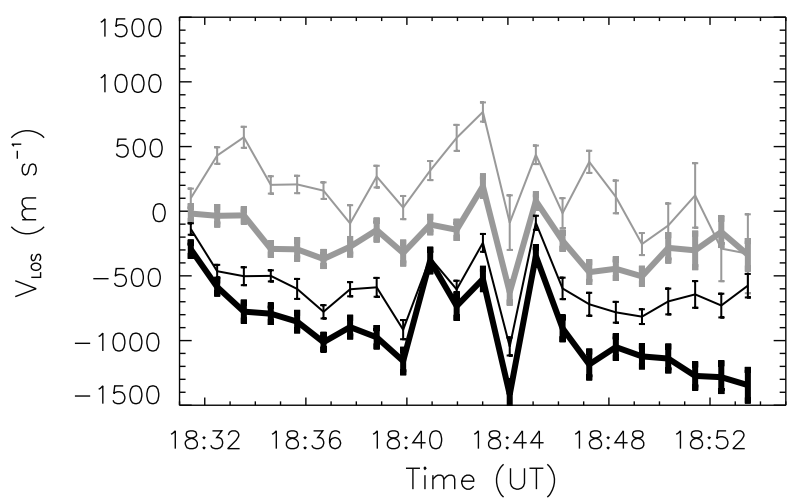

the photospheric velocity of pores as was reported by Hirzberger (2003) and Sobotka et al. (2012).

We investigated a dynamic interaction between the magnetic flux tubes (pores and SMCs) and the photospheric gas motion by using the G-band data taken with Hinode/SOT during our observation. For horizontal gas motion in the photosphere, we first aligned G-band images and applied subsonic-filtering to reduce solar oscillation effect and noise. We then applied the nonlinear affine velocity estimator (NAVE) to the successive G-band images and got a first-order surface velocity field including the first derivatives with respect to the horizontal coordinates as described by Chae and Sakurai (2008). The divergence of the velocity was obtained from those first derivatives given by the NAVE technique. We tracked the position of the pores and SMCs using NAVE, as seen in the G-band movie (electronic supplementary material). Finally, we measured the averaged horizontal velocity and divergence within the radius of $0.5^{\prime \prime}$ around the position of the pores and SMCs. The transverse acceleration was derived from the running difference of the averaged horizontal velocity. The LOS 


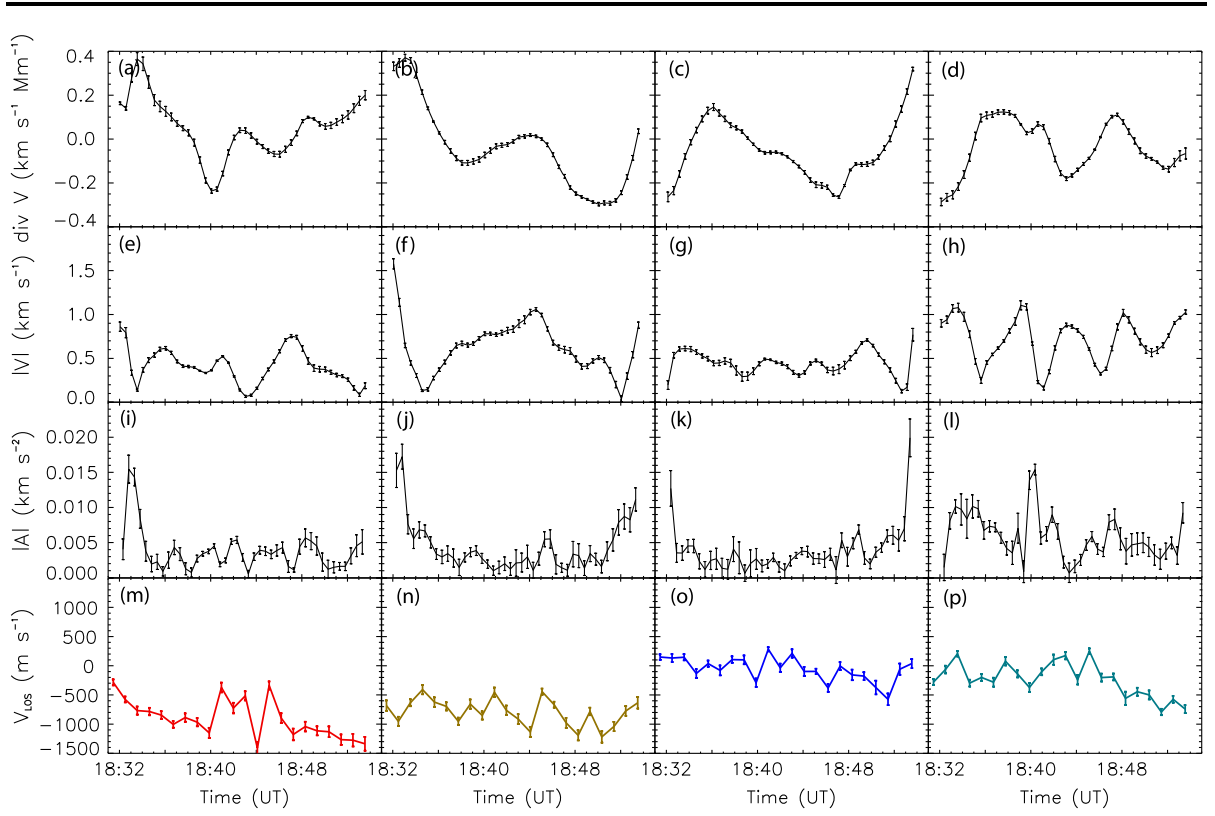

Figure 9 Time variations of $(a-d)$ the divergence of velocity, $(e-h)$ transverse velocity, and (i-1) acceleration at the pores and SMCs, which are estimated from G-band images by applying the NAVE technique. FISS LOS velocities above pores P1 (m), P2 (n), and SMC1 (o) and SMC2 (p) are estimated by applying the bisector method to the wing $(\Delta \lambda=2.35 \AA$ ) spectra of the Ca II $8542 \AA$ line.

velocity in the chromosphere obtained by FISS was similarly averaged inside the pores and SMCs. Figure 9 shows a comparison between the temporal variations of the photospheric gas motion (divergence, transverse velocity, and acceleration) inside the pores and SMCs, and the chromospheric LOS velocity on the pores and SMCs. We found that the shape and brightness of the pores and SMCs observed in the G-band change with time due to their interaction with granular motion, but there is no clear relationship between the physical parameters representing the photospheric gas motion and the chromospheric LOS velocity. No apparent difference in the LOS velocity between the pores and SMCs was found.

The time evolution of pores and SMCs and their interaction with the surrounding gas motions can be thoroughly studied when the structures are stably imaged with high spatial resolution and sensitivity. Unfortunately, the performance of currently working low-order adaptive optics of NST/FISS is not satisfactory in this regard.

\section{Discussion and Conclusion}

We have derived the physical characteristics of small pores based on the simultaneous observation of Hinode/SOT and NST/FISS. To our knowledge, this is the first detailed report on the connection of LOS plasma flows in tiny pores between the photosphere and the lower chromosphere through the determination of the chromospheric LOS velocities in pores and SMCs simultaneously, as well as their photospheric LOS velocities.

Hinode/SOT-SP observation elucidates that the plasma in the pores is always moving down on the photosphere, while the FISS observation of the wing spectra of Ca II $8542 \AA$ and $\mathrm{H} \alpha$ detected upflows at the pore in the low chromosphere. Figure 10 illustrates a schematic 


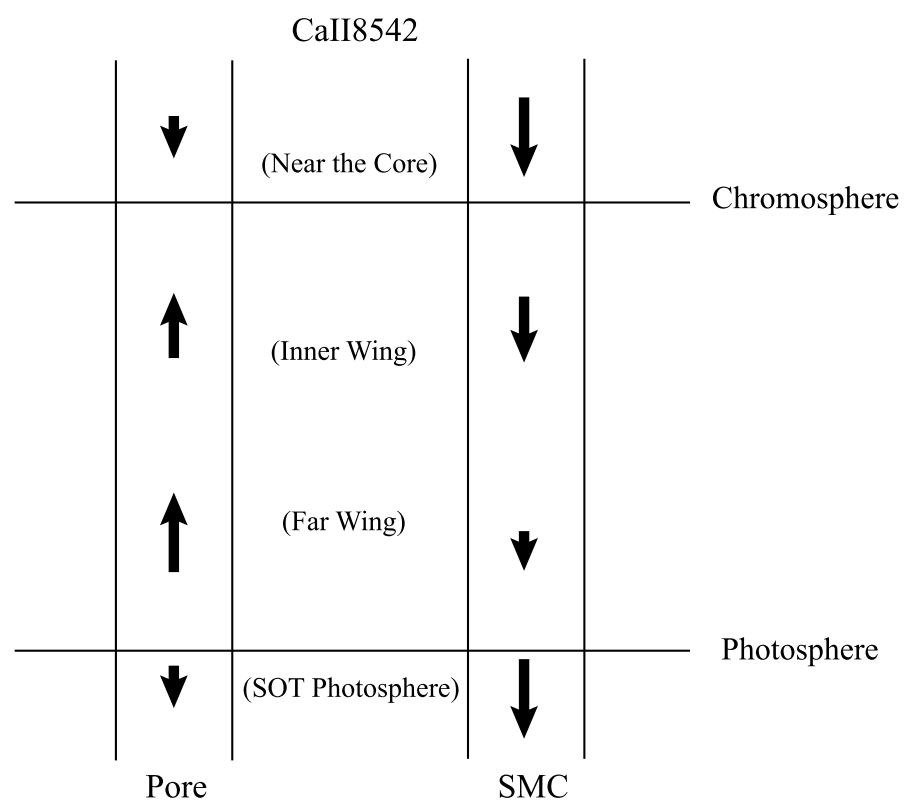

Figure 10 Vertical motion of plasma in the pore and SMC in the photosphere and chromosphere.

picture about the height variation of vertical motion inside pores and in SMCs, respectively. The inspection of the Ca II $8542 \AA$ line from the wing to the core by applying the bisector method revealed that the upflow velocity in the pores decreases with height and turns into downward in the upper chromosphere, while downflows in the SMCs gain their speed in the lower chromosphere. Those lasted at least 20 min during the observational period (Figure 8).

Our observations on the dynamic property of flux tubes could be understood by considering a dynamic interaction between solar magnetic elements and granular convection simulated by Steiner et al. (1998) and Kato et al. (2011). According to their two-dimensional MHD simulation, the interior of a pore cools rapidly and induces strong downflow around the pore owing to radiative losses and suppression of energy transport by convection in the pore. Their simulation predicts that the downflow turns into upflow after interacting with a dense and deep layer under the pore. This theoretical description is well consistent with our observations of LOS velocities in the photosphere and low chromosphere. It is likely that only strong downflow can excite an upward propagating shock wave as predicted by Grossmann-Doerth, Schüssler, and Steiner (1998), and it is expected that the upward shock is continuously observed in the upper photosphere and lower chromosphere.

In SMCs, we detected downflows, but no upflows at all. This might be explained by the absence of localized rapid cooling in SMCs because of the heat coming from the surroundings. In other words, the existence of downflows only suggests that a more or less continuous process may be more important in SMCs than a localized, impulsive cooling process unlike pores. If the downflow at the photosphere is caused by the convective collapse, the time scale of the event is several minutes (e.g. Narayan, 2011). Persistent downflows over 20 min might represent the spatially averaged property of multiple flux tubes which are subject to convective instability. 
In summary, we observed upward-directed dynamic motions at the tiny pores in the lower chromosphere by inspecting the wing spectra of chromospheric lines, which might be related to upwardly propagating magnetoacoustic waves predicted by Steiner et al. (1998) and Kato et al. (2011).

We are expecting that the high-order adaptive optics system for NST will provide stable observations with high spatial/spectral/temporal (10 s) resolutions, which will contribute to understanding the nature of the upward motion in the pores and SMCs, as well as their interaction with ambient plasma.

Acknowledgements We are grateful to the referee for helpful and constructive comments. This work was supported by the "Development of Korea Space Weather Center" of KASI and the KASI basic research funds and by the Korea Research Foundation Grant funded by the Korean Government (KRF-2008-220-C00022, 2011-0028102). One of the authors (K.-S.C.) is very thankful to R.J. Rutten for his valuable comments and to $\mathrm{S}$. Tsuneta for supporting of collaborative visiting in NAOJ, where the part of present work has been carried out. Hinode is a Japanese mission developed and launched by ISAS/JAXA, with NAOJ as domestic partner and NASA and STFC (UK) as international partners. It is operated by these agencies in cooperation with ESA and NSC (Norway).

Open Access This article is distributed under the terms of the Creative Commons Attribution License which permits any use, distribution, and reproduction in any medium, provided the original author(s) and the source are credited.

\section{References}

Ahn, K., Chae, J., Park, H.M., Nah, J., Park, Y.-D., Jang, B.H., Moon, Y.-J.: 2008, J. Korean Astron. Soc. 41, 39.

Cameron, R., Schüssler, M., Vögler, A., Zakharov, V.: 2007, Astron. Astrophys. 474, 261.

Cauzzi, G., Reardon, K., Uitenbroek, H., Cavallini, F., Falchi, A., Falciani, R., Janssen, K., Rimmele, T., Vecchio, A., Wöger, F.: 2008, Astron. Astrophys. 480, 515.

Cauzzi, G., Reardon, K., Rutten, R.J., Tritschler, A., Uitenbroek, H.: 2009, Astron. Astrophys. 503, 577.

Chae, J., Sakurai, T.: 2008, Astrophys. J. 689, 593.

Chae, J., Park, H.M., Ahn, K., Yang, H., Park, Y.-D., Nah, J., Jang, B.H., Cho, K.-S., Cao, W., Goode, P.R.: 2012, Solar Phys. doi:10.1007/s11207-012-0147-x.

Cho, K.-S., Bong, S.-C., Chae, J., Kim, Y.-H., Park, Y.-D.: 2010, Astrophys. J. 723, 440.

Goode, P.R., Denker, C.J., Didkovsky, L.I., Uhn, J.R., Wang, H.: 2003, J. Korean Astron. Soc. 36, S125.

Goode, P.R., Coulter, R., Gorceix, N., Yurchyshyn, V., Cao, W.: 2010, Astron. Nachr. 331, 620.

Grossmann-Doerth, U., Schüssler, M., Steiner, O.: 1998, Astron. Astrophys. 337, 928.

Hirzberger, J.: 2003, Astron. Astrophys. 405, 331.

Kato, Y., Steiner, O., Steffen, M., Suematsu, Y.: 2011, Astrophys. J. Lett. 730, L24.

Keppens, R., Martinez Pillet, V.: 1996, Astron. Astrophys. 4, 291.

Keil, S.L., Balasubramaniam, K.S., Smaldone, L.A., Reger, B.: 1999, Astrophys. J. 510, 422.

Knölker, M., Schüssler, M.: 1988, Astron. Astrophys. 202, 275.

Kosugi, T., Matsuzaki, K., Sakao, T., Shimizu, T., Sone, Y., Tachikawa, S., et al.: 2007, Solar Phys. $243,3$.

Leenaarts, J., Rutten, R.J., Carlsson, M., Uitenbroek, H.: 2006, Astron. Astrophys. 452, L15.

Martinez Pillet, V., Lites, B.W., Skumanich, A.: 1997, Astrophys. J. 474, 810.

Morinaga, S., Sakurai, T., Ichimoto, K., Yokoyama, T., Shimojo, M., Katsukawa, Y.: 2008, Astron. Astrophys. 481, L29.

Nagata, S., Tsuneta, S., Suematsu, Y., Ichimoto, K., Katsukawa, Y., Shimizu, T., et al.: 2008, Astrophys. J. Lett. 677, L145.

Narayan, G.: 2011, Astron. Astrophys. 529, A79.

Parker, E.N.: 1979, Astrophys. J. 234, 333.

Pesnell, W.D., Thompson, B.T., Chamberlin, P.C.: 2011, Solar Phys. 275, 3.

Rees, D.E., Semel, M.D.: 1979, Astron. Astrophys. 74, 1.

Schou, J., Scherrer, P.H., Bush, R.I., Wachter, R., Couvidat, S., Rabello-Soares, M.C., et al.: 2012, Solar Phys. 275, 229.

Skumanichi, A., Lites, B.W.: 1987, Astrophys. J. 322, 473.

Sobotka, M., Del Moro, D., Jurčák, J., Berrilli, F.: 2012, Astron. Astrophys. 537, A85. 
Spruit, H.C.: 1979, Solar Phys. 61, 363.

Spruit, H.C., Zwaan, C.: 1981, Solar Phys. 70, 207.

Steiner, O., Grossmann-Doerth, U., Knoelker, M., Schuessler, M.: 1998, Astrophys. J. 495, 468.

Tsuneta, S., Ichimoto, K., Katsukawa, Y., Lites, B.W., Matsuzaki, K., Nagata, S., et al.: 2008, Solar Phys. 249, 167.

Uitenbroek, K.H.: 2006, Astrophys. J. 639, 516. 